\title{
Nitrogen Recovery from Enhanced Efficiency Fertilizers and Urea in Intensively Managed Black Walnut (Juglans nigra) Plantations
}

\author{
Joshua L. Sloan ${ }^{1,+}$, Francis K. Salifu ${ }^{2}$ and Douglass F. Jacobs $1, *$ (D) \\ 1 Hardwood Tree Improvement and Regeneration Center, Department of Forestry and Natural Resources, \\ Purdue University, West Lafayette, IN 47907, USA; jlsloan@nmhu.edu \\ 2 Salifu Environmental Solutions, Calgary, AB T3P0G2, Canada; kuaaka@yahoo.com \\ * Correspondence: djacobs@purdue.edu \\ + Present address: Department of Forestry, New Mexico Highlands University, Las Vegas, NM 87701, USA.
}

check for updates

Citation: Sloan, J.L.; Salifu, F.K.; Jacobs, D.F. Nitrogen Recovery from Enhanced Efficiency Fertilizers and Urea in Intensively Managed Black Walnut (Juglans nigra) Plantations. Forests 2021, 12, 352. https:// doi.org/10.3390/f12030352

Academic Editor: Nelson Thiffault

Received: 28 January 2021

Accepted: 11 March 2021

Published: 17 March 2021

Publisher's Note: MDPI stays neutral with regard to jurisdictional claims in published maps and institutional affiliations.

Copyright: (c) 2021 by the authors. Licensee MDPI, Basel, Switzerland. This article is an open access article distributed under the terms and conditions of the Creative Commons Attribution (CC BY) license (https:// creativecommons.org/licenses/by/ $4.0 /)$.

\begin{abstract}
Intensively managed forest plantations often require fertilization to maintain site fertility and to improve growth and yield over successive rotations. We applied urea-based "enhancedefficiency fertilizers" (EEF) containing 0.5 atom $\%{ }^{15} \mathrm{~N}$ at a rate of $224 \mathrm{~kg} \mathrm{~N}^{-1}$ to soils under midrotation black walnut (Juglans nigra L.) plantations to track the fate of applied ${ }^{15} \mathrm{~N}$ within aboveground ecosystem components during the 12-month period after application. Treatments included Agrotain Ultra (urea coated with a urease inhibitor), Arborite EC (urea coated with water-soluble boron and phosphate), Agrium ESN (polymer-coated urea), uncoated urea, and an unfertilized control. Agrotain Ultra and Arborite EC increased N concentrations of competing vegetation within one month after fertilization, while neither Agrium ESN nor uncoated urea had any effect on competing vegetation $\mathrm{N}$ concentrations during the experiment. Agrotain Ultra and Arborite EC increased $\delta^{15} \mathrm{~N}$ values in leaves of crop trees above those of controls at one and two months after fertilization, respectively. By contrast, Agrium ESN and uncoated urea had no effect on $\delta^{15} \mathrm{~N}$ values in leaves of crop trees until three months after fertilization. Fertilizer N recovery (FNR) varied among ecosystem components, with competing vegetation acting as a sink for applied nutrients. There were no significant differences in FNR for all the urea-based EEF products compared to uncoated urea. Agrium ESN was the only EEF that exhibited controlled-release activity in this study, with other fertilizers behaving similarly to uncoated urea.
\end{abstract}

Keywords: nutrient cycling; plantation productivity; plant growth; competing vegetation; fertilizer $\mathrm{N}$ recovery; fertilization; black walnut; $\delta^{15} \mathrm{~N}$

\section{Introduction}

Black walnut (Juglans nigra L.) is the highest value tree species in the continental United States [1], and it typically occurs as a minor component of mixed deciduous forests in central and eastern parts of the U.S. [2]. The demand for high quality hardwoods, such as black walnut, that are used in fine furniture, cabinetry, gunstocks, paneling, and specialty crafts has been increasing dramatically. Due to its high value, black walnut has been routinely harvested from natural forests, causing depletions in the supply of quality trees. As a result, there has been considerable interest in recent decades in the establishment and intensive management of black walnut in plantations [3,4]. Although only about 5585 ha of black walnut plantations exist in the U.S. (representing about $1 \%$ of total black walnut cubic foot volume in the U.S.) [5], plantation area is rapidly increasing. In Indiana, which is centrally located within black walnut's native range, 2400-3200 ha of trees are planted every year and black walnut represents about $18 \%$ of planted trees [6].

Based on the high nutrient demand, rapid growth potential, and extraordinary value of black walnut timber, some industrial black walnut plantation managers in Indiana and Spain have been operationally fertilizing stands every year following planting with the 
intention of significantly promoting growth and reducing rotation ages [7-9]. Although several studies have shown improved plantation productivity with fertilization in both black walnut and other stand types [10,11], others have demonstrated low fertilizer nitrogen use efficiency (FNUE) and low productivity [12]. To address low FNUE and low productivity issues, various "enhanced-efficiency fertilizers" (EEF) have been developed to reduce N loss, increase $\mathrm{N}$ availability, improve fertilizer $\mathrm{N}$ recovery (FNR), and enhance plantation growth and yield [11,13]. The EEFs can be divided into slow release (SRN), controlled release (CRN), and stabilized (SNF) $\mathrm{N}$ fertilizers [13]. The SRN products slowly release fertilizer $\mathrm{N}$ due to microbial decomposition [14]. The CRN products have coatings around the fertilizer $\mathrm{N}$ to alter the rate, pattern, and duration of fertilizer $\mathrm{N}$ release $[14,15]$. The SNF products have compounds to inhibit rapid fertilizer $\mathrm{N}$ transformation to less stable forms [16]. The different attributes of the various $\mathrm{N}$ containing EEF products increase the options for $\mathrm{N}$ fertilization under diverse conditions to help optimize plant $\mathrm{N}$ uptake and increase FNUE compared to urea.

However, although fertilization has been found to reduce nutrient limitations and improve plantation productivity and performance, it may also result in negative impacts such as stimulating the growth of non-target vegetation or increasing the offsite movement of nutrients through losses due to volatilization, runoff, or leaching. These factors and related uncertainties lead to the need to improve our understanding of fertilizer fate and use efficiency in plantation settings in order to limit nutrient losses and improve plantation productivity. The goal of this study was to characterize $\mathrm{N}$ uptake and partitioning within aboveground ecosystem components and to determine whether FNR was greater for EEFs compared to urea. To accomplish this, we compared fertilizer $\mathrm{N}$ uptake in midrotation black walnut plantations following fertilization for three urea-based EEF products, uncoated urea, and an unfertilized control to determine if there were differences among treatments within aboveground ecosystem components for: (1) $\mathrm{N}$ concentrations; (2) $\delta^{15} \mathrm{~N}$ levels to quantify the incorporation of applied N; and (3) FNR and partitioning.

\section{Materials and Methods}

\subsection{Site Description}

In July 2010, eight non-contiguous areas were selected in a privately-owned midrotation black walnut plantation with a total area of about 50 ha (centered at approximately $40.32877^{\circ} \mathrm{N}, 86.70805^{\circ} \mathrm{W}$ ) in Sheffield Township, Tippecanoe County, IN, USA. Slopes in the plantation were under $5 \%$ with the majority of soils underlying the plantation being well-drained to excessively drained. Soils under the plantations included Hononegah fine sandy loam (37.3\% of the land area), Ockley silt loam (10.4\% of the land area), FincastleCrosby complex (9.7\% of the land area), Sleeth loam ( $8.2 \%$ of the land area), Cohoctah fine sandy loam (7.3\% of the land area), Ouiatenon sandy loam (7.3\% of the land area), Mahalasville silty clay loam (7.1\% of the land area), and various minor components.

Each selected area (about 6 ha each) served as a block, with areas selected to spatially represent the entire plantation. The plantation was established in 1989 with a tree spacing of $6.9 \mathrm{~m}$ between rows and $3.0 \mathrm{~m}$ between trees within rows. Within each block, five dominant crop trees were selected for inclusion in the study. Crop trees were selected to spatially represent the entirety of the block, with sufficient space between trees to prevent cross-contamination from the application of ${ }^{15} \mathrm{~N}$-enriched fertilizers. Crop trees selected for the study had an average diameter at breast height ( \pm standard deviation) of $24.63 \pm 2.69$ $\mathrm{cm}$, average total height of $15.19 \pm 1.67 \mathrm{~m}$, average live crown length of $8.73 \pm 1.80 \mathrm{~m}$, and the average live crown ratio was $0.58 \pm 0.10$. At the time of selection, each crop tree in each block was randomly assigned to one of five fertilizer treatments (described below).

The understory of the plantation was relatively homogeneous and was populated by a mixture of woody and herbaceous species, with the majority of understory species present being herbaceous. The main woody species among the competing vegetation was black raspberry (Rubus occidentalis L.), while the herbaceous species composition varied seasonally and consisted of various members of the Poaceae, Asteraceae, and Brassicaceae, 
with small numbers of individuals from other families. Historical control of competing vegetation in this stand prior to the study consisted mainly of mechanical control via mowing. The vegetation control history and methods in this stand, as well as the density and composition of vegetative competition observed, can be considered representative for black walnut plantations in this region.

\subsection{Fertilizer Treatments and ${ }^{15} \mathrm{~N}$ Labeling}

The five fertilizer treatments consisted of an unfertilized control, Agrotain Ultra (Agrotain), Arborite EC (Arborite), Agrium ESN (ESN), and urea. All fertilizers were enriched to 0.5 atom $\%{ }^{15} \mathrm{~N}$ during manufacture, except for the unfertilized control treatment which served as both an isotopic and fertilization control throughout the study. All fertilizers were urea-based, with Agrotain, Arborite, and ESN serving as examples of urea-based enhanced-efficiency fertilizers (EEF) which employ different mechanisms for controlling release rates. The urea applied in the Agrotain Ultra treatment was an SNF coated with a urease inhibitor, $\mathrm{N}$-(n-butyl) thiophosphoric triamide (NBPT), to reduce ammonia volatilization from applied urea (Koch Agronomic Services, LLC, Wichita, KS, USA). The urea applied in the Arborite EC treatment was an SRN coated with watersoluble boron and phosphate using a proprietary process in order to regulate rates of $\mathrm{N}$ release and loss from fertilizer granules (Weyerhaeuser Company, Seattle, DC, USA). The urea applied in the Agrium ESN treatment was a CRN contained within a polymer coating which moderates $\mathrm{N}$ release rates in proportion to soil temperature (Agrium, Inc., Calgary, AB, Canada). All fertilizer applications were surface broadcast by hand at a rate of $224 \mathrm{~kg} \mathrm{~N} \mathrm{ha}^{-1}$ within a $100 \mathrm{~m}^{2}$ circular plot centered on each selected crop tree. Each fertilized tree received $22.4 \mathrm{~kg} \mathrm{~N}$ via its assigned fertilizer treatment except crop trees assigned to the control treatment. For logistical reasons, the experiment was installed and executed in two stages, with fertilizer treatments being applied to blocks 1-4 in May 2011 and to blocks 5-8 in May 2012. Initial pre-fertilization time zero sampling and postfertilization sampling of crop tree and ecosystem components are described below.

\subsection{Sample Collection}

Black walnut crop tree leaves were collected from the middle one-third of the south side of each crop tree selected for inclusion in the study prior to application of fertilizer treatments and at intervals during the first growing season after fertilization. At each sampling time, 10 complete leaves (i.e., leaflets and rachis) were collected from each crop tree by hand using an aerial lift (Altec, Inc., Birmingham, AL, USA), with each leaf serving as a sub-sample. Initial pre-fertilization (time zero) leaves were collected in July of the year prior to fertilization (i.e., July 2010 for blocks 1-4 and July 2011 for blocks 5-8) due to the absence of crop tree leaves at the time of fertilization. After fertilization, leaves were collected from each black walnut crop tree as described above in June (one month after fertilization), July (two months after fertilization), and August (three months after fertilization) in the year of fertilization (i.e., 2011 for blocks 1-4 and 2012 for blocks 5-8). Upon collection, each leaf was placed in a labeled paper bag before placement in a cooler for immediate transport to the Forest Ecology, Soils, and Silviculture Laboratory (FESSL) in the Department of Forestry and Natural Resources at Purdue University (FESSL, West Lafayette, IN, USA) for processing.

Competing vegetation and forest floor litter (i.e., the Oi horizon) were collected from four $0.0625 \mathrm{~m}^{2}(25 \mathrm{~cm} \times 25 \mathrm{~cm})$ sub-sampling areas located approximately $2.82 \mathrm{~m}$ to the $\mathrm{NE}, \mathrm{SE}, \mathrm{SW}$, and NW of each crop tree prior to application of fertilizer treatments and at intervals during the first year after fertilization. At each sampling time, all living vegetation emerging from the soil within each sub-sampling area was hand-cut at ground level and placed in a labeled paper bag before placement in a cooler for immediate transport to FESSL for processing. All forest floor litter of the Oi horizon was then collected from within each sub-sampling area and treated as described for the competing vegetation. Sub-sampling areas were randomly spatially shifted slightly at each sampling time so as to avoid re- 
sampling areas during the course of the study. Initial pre-fertilization (time zero) sampling of competing vegetation and litter occurred immediately prior to fertilization (i.e., May 2011 for blocks 1-4 and May 2012 for blocks 5-8). After fertilization, competing vegetation and litter were collected from sub-sampling areas surrounding each crop tree in June (one month after fertilization), July (two months after fertilization), August (three months after fertilization), November (six months after fertilization), and May of the following year (12 months after fertilization), with this sampling scheme initiated in 2011 for blocks 1-4 and in 2012 for blocks 5-8.

\subsection{Sample Preparation and Analyses}

Upon arrival at FESSL, leaf areas of all black walnut crop tree leaf sub-samples were immediately measured using an LI-3000 portable leaf area meter (LI-COR, Inc., Lincoln, NE, USA). Sub-samples of plant-based ecosystem components (i.e., black walnut crop tree leaves, competing vegetation, and litter) were air-dried to constant mass and dry masses were recorded before being ground in a Wiley mill (Thomas Scientific, Swedesboro, NJ, USA) to pass a 20-mesh screen. Subsequently, all ground sub-samples for each plant-based ecosystem component $\times$ fertilizer treatment $\times$ sampling time $\times$ block combination were composited, homogenized, and submitted to the Department of Forest Resources and Environmental Conservation at Virginia Tech for determination of $\mathrm{N}$ concentration and $\delta^{15} \mathrm{~N}$ using a coupled elemental analysis isotope ratio mass spectrometer (IsoPrime 100 EA-IRMS, Isoprime, Ltd., Manchester, UK; [11]).

\subsection{Calculations and Statistical Analyses}

For all sampled ecosystem components (i.e., black walnut crop tree leaves, competing vegetation, and litter), all dependent variables measured and reported in Tables 1-7 were directly quantified, except fertilizer $\mathrm{N}$ recovery (FNR). For estimation of FNR in leaves of black walnut crop trees, total leaf area contained within the canopy of each crop tree was estimated using the allometric relationships described by [17]. The equation employed was:

$$
\mathrm{A}=2.734 \times \mathrm{DBH}^{1.487} \times \mathrm{LCR}^{1.519}
$$

where: A = the estimated total leaf area $\left(\mathrm{m}^{2}\right)$ of a given black walnut crop tree; $\mathrm{DBH}=$ the diameter at breast height $(\mathrm{cm})$ of the black walnut crop tree; and LCR = the live crown ratio of the black walnut crop tree determined by dividing the length of live crown $(\mathrm{m})$ of the black walnut crop tree by its total height $(\mathrm{m})$. After total leaf areas were estimated for each black walnut crop tree, the FNR in leaves of each black walnut crop tree canopy was estimated as follows.

First, for leaves of each crop tree at each sampling time, leaf $\mathrm{N}$ content was calculated by multiplying the average dry mass of sub-sampled leaves by the measured $\mathrm{N}$ concentration (\%, on a dry mass basis). Second, reported $\delta^{15} \mathrm{~N}$ values for each crop tree leaf sample at each sampling time were mathematically reverted to ${ }^{15} \mathrm{~N}:{ }^{14} \mathrm{~N}$ values via simple rearrangement of the equation used for calculating $\delta^{15} \mathrm{~N}$. Third, total mass of ${ }^{15} \mathrm{~N}$ in each crop tree leaf sample was determined based upon the calculated leaf $\mathrm{N}$ content and ${ }^{15} \mathrm{~N}:{ }^{14} \mathrm{~N}$ of each sample. Fourth, the calculated total mass of ${ }^{15} \mathrm{~N}$ in each crop tree leaf sample was divided by the average area of leaf sub-samples for each crop tree at the corresponding sampling time to yield total mass of ${ }^{15} \mathrm{~N}$ per unit leaf area for each crop tree at each sampling time. Fifth, values for total mass of ${ }^{15} \mathrm{~N}$ per unit leaf area of unfertilized control trees were assumed to be representative of ${ }^{15} \mathrm{~N}$ natural abundance in crop tree leaves; consequently, these values were averaged for each sampling time and subtracted from the calculated total mass of ${ }^{15} \mathrm{~N}$ per unit leaf area for each fertilized crop tree at each sampling time to yield an estimate of the mass of fertilizer-derived ${ }^{15} \mathrm{~N}$ per unit leaf area for each fertilized crop tree at each sampling time. Sixth, the estimated mass of fertilizer-derived ${ }^{15} \mathrm{~N}$ per unit leaf area for each fertilized crop tree at each sampling time was multiplied by the estimated total leaf area of each fertilized crop tree to produce an estimate of the mass of fertilizer-derived ${ }^{15} \mathrm{~N}$ present in the canopy of each fertilized crop tree at each sampling time. Finally, the 
estimated mass of fertilizer-derived ${ }^{15} \mathrm{~N}$ present in the canopy of each fertilized crop tree at each sampling time was divided by the mass of ${ }^{15} \mathrm{~N}$ initially applied via each fertilizer treatment and expressed as a percentage to produce an estimate of FNR from the leaves of each fertilized black walnut crop tree at each sampling time.

Table 1. ANOVA results for plant-related response variables. For each entry, $p$-values are displayed for the overall model, main effects, and all main effect interactions. Values in bold were considered significant at $p<0.05$. No main effects or interactions were considered significant in the absence of overall model significance. Abbreviations: Fert $=$ fertilizer treatment; Time $=$ time relative to fertilization.

\begin{tabular}{|c|c|c|c|c|c|}
\hline $\begin{array}{l}\text { Ecosystem } \\
\text { Component }\end{array}$ & Dependent Variable & Model & Fert & Time & Fert $\times$ Time \\
\hline \multirow{6}{*}{ Crop tree } & Leaf dry mass & 0.0107 & 0.0384 & $<0.0001$ & 0.9893 \\
\hline & Leaf area & $<0.0001$ & 0.0710 & $<0.0001$ & 0.9481 \\
\hline & Leaf $\mathrm{N}$ concentration & 0.0067 & 0.0301 & $<0.0001$ & 0.8678 \\
\hline & Leaf $\mathrm{N}$ content & 0.0908 & 0.0071 & 0.1082 & 0.7919 \\
\hline & $\delta^{15} \mathrm{~N}$ of leaves & $<0.0001$ & $<0.0001$ & $<0.0001$ & 0.0026 \\
\hline & Fertilizer N recovery & 0.6996 & 0.2100 & 0.6472 & 0.8554 \\
\hline \multirow{5}{*}{$\begin{array}{l}\text { Competing } \\
\text { vegetation }\end{array}$} & Dry mass & $<0.0001$ & 0.6466 & $<0.0001$ & 0.8941 \\
\hline & $\mathrm{N}$ concentration & $<0.0001$ & 0.0020 & $<0.0001$ & 0.0112 \\
\hline & $\mathrm{N}$ content & $<0.0001$ & 0.0751 & $<0.0001$ & 0.0684 \\
\hline & $\delta^{15} \mathrm{~N}$ & $<0.0001$ & $<0.0001$ & $<0.0001$ & $<0.0001$ \\
\hline & Fertilizer N recovery & $<0.0001$ & 0.8375 & $<0.0001$ & 0.2000 \\
\hline \multirow{5}{*}{ Litter } & Dry mass & 0.0041 & 0.9419 & $<0.0001$ & 1.0000 \\
\hline & $\mathrm{N}$ concentration & 0.0655 & 0.0022 & 0.0130 & 0.9223 \\
\hline & $\mathrm{N}$ content & 0.0108 & 0.7472 & $<0.0001$ & 1.0000 \\
\hline & $\delta^{15} \mathrm{~N}$ & $<0.0001$ & $<0.0001$ & $<0.0001$ & $<0.0001$ \\
\hline & Fertilizer N recovery & 0.9112 & 0.7840 & 0.2000 & 0.9968 \\
\hline
\end{tabular}

Table 2. Mean values ( \pm standard error of the mean) for plant-related response variables for which no interaction was observed, by fertilization treatment. For crop tree variables, values displayed are fertilizer treatment means $(n=8)$. For competing vegetation and litter variables, values displayed are fertilizer treatment means $(n=8)$, with each sample being represented by four $0.0625 \mathrm{~m}^{2}$ sub-sampling areas surrounding each crop tree from which all vegetation and litter was collected at each sampling time. Fertilizer $\mathrm{N}$ recovery values are the percentage of fertilizer-derived ${ }^{15} \mathrm{~N}$ recovered from the specified component in each fertilizer treatment relative to the amount initially applied.

\begin{tabular}{|c|c|c|c|c|c|c|}
\hline \multirow{2}{*}{$\begin{array}{l}\text { Ecosystem } \\
\text { Component }\end{array}$} & \multirow{2}{*}{ Dependent Variable } & \multicolumn{5}{|c|}{ Fertilizer Treatment } \\
\hline & & Control & Agrotain & Arborite & ESN & Urea \\
\hline \multirow{5}{*}{ Crop tree } & Leaf dry mass $\left(\mathrm{g} \mathrm{leaf}^{-1}\right)^{*}$ & $1.89(0.11) \mathrm{AB}$ & $1.68(0.09) \mathrm{B}$ & $2.08(0.10) \mathrm{A}$ & $1.79(0.11) \mathrm{AB}$ & $1.78(0.09) \mathrm{AB}$ \\
\hline & Leaf area $\left(\mathrm{cm}^{2} \mathrm{leaf}^{-1}\right)$ & $202.51(10.22)$ & $189.51(8.38)$ & $218.38(10.04)$ & $196.83(12.23)$ & $184.95(7.81)$ \\
\hline & Leaf $\mathrm{N}$ concentration $(\%) *$ & $1.77(0.05) \mathrm{B}$ & $2.03(0.07) \mathrm{AB}$ & $2.03(0.07) \mathrm{A}$ & $1.91(0.07) \mathrm{AB}$ & $1.90(0.06) \mathrm{AB}$ \\
\hline & Leaf $\mathrm{N}$ content $\left(\mathrm{g}\right.$ leaf ${ }^{-1}$ ) & $0.03(0.00)$ & $0.03(0.00)$ & $0.04(0.00)$ & $0.03(0.00)$ & $0.03(0.00)$ \\
\hline & Fertilizer N recovery (\%) & - & $1.78(0.52)$ & $2.66(0.62)$ & $1.01(0.44)$ & $1.70(0.50)$ \\
\hline \multirow{3}{*}{$\begin{array}{l}\text { Competing } \\
\text { vegetation }\end{array}$} & Dry mass (g sample ${ }^{-1}$ ) & $9.53(0.72)$ & $10.05(0.95)$ & $10.57(1.05)$ & $10.75(0.77)$ & $10.85(0.98)$ \\
\hline & $\mathrm{N}$ content (g sample ${ }^{-1}$ ) & $0.15(0.01)$ & $0.20(0.02)$ & $0.22(0.02)$ & $0.21(0.02)$ & $0.22(0.03)$ \\
\hline & Fertilizer N recovery (\%) & 一 & $5.39(1.56)$ & $5.86(1.70)$ & $5.17(1.14)$ & $6.73(1.84)$ \\
\hline \multirow{4}{*}{ Litter } & Dry mass (g sample ${ }^{-1}$ ) & $9.10(1.50)$ & $9.54(1.64)$ & $7.85(1.24)$ & $8.85(1.38)$ & $9.85(1.88)$ \\
\hline & $\mathrm{N}$ concentration $(\%)$ & $0.94(0.03)$ & $1.20(0.05)$ & $1.10(0.05)$ & $1.08(0.05)$ & $1.15(0.04)$ \\
\hline & $\mathrm{N}$ content (g sample ${ }^{-1}$ ) & $0.09(0.02)$ & $0.12(0.02)$ & $0.09(0.02)$ & $0.10(0.02)$ & $0.12(0.02)$ \\
\hline & Fertilizer $\mathrm{N}$ recovery $(\%)$ & - & $2.42(1.12)$ & $0.54(0.76)$ & $1.14(0.89)$ & $2.44(1.61)$ \\
\hline
\end{tabular}

Variables marked with an * showed a significant fertilizer treatment main effect $(p<0.05)$. For a given variable, means followed by different letters differed significantly $(\alpha=0.05)$. - indicates that no values exist for this entry. 
Table 3. Mean values ( \pm standard error of the mean) for plant-related response variables for which no interaction was observed, by time. For crop tree variables, values displayed are means by sampling time (i.e., averaged across treatments for a given time; $n=8)$. For competing vegetation and litter variables, values displayed are means by sampling time $(n=8)$, with each sample being represented by four $0.0625 \mathrm{~m}^{2}$ sub-sampling areas surrounding each crop tree from which all vegetation and litter was collected. Fertilizer $\mathrm{N}$ recovery values are the percentage of fertilizer-derived ${ }^{15} \mathrm{~N}$ recovered from the specified component at each sampling time relative to the amount initially applied.

\begin{tabular}{|c|c|c|c|c|c|c|c|}
\hline \multirow{2}{*}{$\begin{array}{l}\text { Ecosystem } \\
\text { Component }\end{array}$} & \multirow{2}{*}{ Dependent Variable } & \multicolumn{6}{|c|}{ Time (Months) } \\
\hline & & 0 & 1 & 2 & 3 & 6 & 12 \\
\hline \multirow{5}{*}{ Crop tree } & Leaf dry mass $\left(\mathrm{g} \mathrm{leaf}^{-1}\right)^{*}$ & $2.10(0.12) \mathrm{A}$ & $1.53(0.08) \mathrm{B}$ & $1.81(0.06) \mathrm{AB}$ & $1.95(0.08) \mathrm{A}$ & - & - \\
\hline & Leaf area $\left(\mathrm{cm}^{2} \text { leaf }^{-1}\right)^{*}$ & $233.56(9.48) \mathrm{A}$ & $158.27(6.90) \mathrm{C}$ & $197.98(5.43) \mathrm{B}$ & 204.17 (8.94) B & - & - \\
\hline & Leaf $\mathrm{N}$ concentration $(\%)$ * & $1.79(0.04) \mathrm{B}$ & $2.14(0.08) \mathrm{A}$ & $1.98(0.06) \mathrm{AB}$ & $1.81(0.04) \mathrm{B}$ & - & - \\
\hline & Leaf $\mathrm{N}$ content $\left(\mathrm{g} \mathrm{leaf}^{-1}\right)$ & $0.04(0.00)$ & $0.03(0.00)$ & $0.04(0.00)$ & $0.04(0.00)$ & - & - \\
\hline & Fertilizer N recovery (\%) & - & $2.10(0.58)$ & $1.49(0.41)$ & $1.80(0.36)$ & - & - \\
\hline \multirow{3}{*}{$\begin{array}{l}\text { Competing } \\
\text { vegetation }\end{array}$} & Dry mass $\left(\text { g sample }^{-1}\right)^{*}$ & $12.48(1.02) \mathrm{AB}$ & $14.16(0.87) \mathrm{A}$ & $9.62(0.66) \mathrm{BC}$ & $9.11(0.76) \mathrm{C}$ & $5.23(0.71) \mathrm{D}$ & $11.80(0.95) \mathrm{ABC}$ \\
\hline & $\mathrm{N}$ content $\left(\mathrm{g} \mathrm{sample}^{-1}\right) *$ & $0.20(0.01) \mathrm{B}$ & $0.37(0.03) \mathrm{A}$ & $0.19(0.01) \mathrm{B}$ & $0.18(0.02) \mathrm{B}$ & $0.08(0.01) \mathrm{C}$ & $0.16(0.01) \mathrm{BC}$ \\
\hline & Fertilizer $\mathrm{N}$ recovery $(\%)$ * & - & $13.76(2.49) \mathrm{A}$ & $6.22(0.94) \mathrm{B}$ & 4.95 (1.14) BC & $-0.10(0.58) \mathrm{C}$ & $2.29(0.75) \mathrm{BC}$ \\
\hline \multirow{4}{*}{ Litter } & Dry mass $\left(\mathrm{g} \mathrm{sample}^{-1}\right)^{*}$ & $11.42(1.19) \mathrm{A}$ & $1.91(0.23) \mathrm{B}$ & $3.30(0.33) \mathrm{B}$ & $3.63(0.37) \mathrm{B}$ & $13.93(1.85) \mathrm{A}$ & $12.50(1.50) \mathrm{A}$ \\
\hline & $\mathrm{N}$ concentration $(\%)$ & $1.13(0.04)$ & $0.91(0.06)$ & $1.14(0.07)$ & $1.11(0.06)$ & $1.16(0.04)$ & $1.00(0.02)$ \\
\hline & $\mathrm{N}$ content $\left(\mathrm{g} \mathrm{sample}^{-1}\right)^{*}$ & $0.13(0.02) \mathrm{A}$ & $0.02(0.00) \mathrm{C}$ & $0.04(0.00) \mathrm{BC}$ & $0.04(0.00) \mathrm{BC}$ & $0.17(0.02) \mathrm{A}$ & $0.12(0.01) \mathrm{AB}$ \\
\hline & Fertilizer N recovery (\%) & - & $-0.02(0.18)$ & $0.98(0.22)$ & $0.60(0.33)$ & $4.01(1.82)$ & $0.98(0.90)$ \\
\hline
\end{tabular}

Variables marked with an * showed a significant main effect for time elapsed after fertilization $(p<0.05)$. For a given variable, means followed by different letters differed significantly $(\alpha=0.05)$. - indicates that no values exist for this entry.

Table 4. Responses of $\delta^{15} \mathrm{~N}(\%)$ of black walnut crop tree leaves to fertilization treatments and time. Table entries are means ( \pm standard error of the mean) for each fertilizer treatment $\times$ time combination $(n=8)$. Means throughout the table marked with the same letters did not differ significantly $(\alpha=0.05)$.

Fertilizer Treatment

\begin{tabular}{ccccc}
\cline { 2 - 5 } Fertilizer Treatment & $\mathbf{0}$ & $\mathbf{2}$ & $\mathbf{3}$ \\
\hline Control & $0.50(0.49) \mathrm{B}$ & $0.46(0.64) \mathrm{B}$ & $0.24(0.38) \mathrm{B}$ & $0.67(0.42) \mathrm{B}$ \\
Agrotain & $0.00(0.38) \mathrm{B}$ & $65.39(20.11) \mathrm{A}$ & $73.12(16.45) \mathrm{A}$ & $84.16(13.66) \mathrm{A}$ \\
Arborite & $-0.12(0.61) \mathrm{B}$ & $48.04(14.04) \mathrm{AB}$ & $68.68(11.72) \mathrm{A}$ & $74.22(9.01) \mathrm{A}$ \\
ESN & $3.38(3.38) \mathrm{B}$ & $9.23(4.38) \mathrm{B}$ & $45.59(14.80) \mathrm{AB}$ & $77.14(12.07) \mathrm{A}$ \\
Urea & $-0.01(0.26) \mathrm{B}$ & $45.71(14.54) \mathrm{AB}$ & $54.47(14.34) \mathrm{AB}$ & $65.72(12.69) \mathrm{A}$ \\
\hline
\end{tabular}

\section{Time (Months)}

Table 5. Competing vegetation $\mathrm{N}$ concentration (\%) in response to fertilizer treatments and time. Table entries are means ( \pm standard error of the mean) for each fertilizer treatment $\times$ time combination $(n=8)$. Means throughout the table marked with the same letters did not differ significantly $(\alpha=0.05)$.

\begin{tabular}{|c|c|c|c|c|c|c|}
\hline \multirow{2}{*}{$\begin{array}{l}\text { Fertilizer } \\
\text { Treatment }\end{array}$} & \multicolumn{6}{|c|}{ Time (Months) } \\
\hline & 0 & 1 & 2 & 3 & 6 & 12 \\
\hline Control & 1.74 (0.19) CDE & $1.78(0.18) \mathrm{CDE}$ & $1.50(0.18) \mathrm{E}$ & $1.54(0.15) \mathrm{DE}$ & $1.73(0.13) \mathrm{CDE}$ & $1.32(0.08) \mathrm{E}$ \\
\hline Agrotain & $1.53(0.17) \mathrm{E}$ & $2.97(0.31) \mathrm{AB}$ & $2.28(0.22) \mathrm{ABCDE}$ & 1.94 (0.15) BCDE & 1.70 (0.17) CDE & 1.65 (0.08) BCDE \\
\hline Arborite & 1.65 (0.14) CDE & $3.01(0.23) \mathrm{A}$ & 2.35 (0.19) ABCDE & $1.92(0.04) \mathrm{CDE}$ & 1.77 (0.21) CDE & 1.44 (0.17) CE \\
\hline ESN & $1.78(0.26) \mathrm{CDE}$ & 2.27 (0.30) ABCDE & $2.20(0.22) \mathrm{ABCDE}$ & $2.53(0.24) \mathrm{ABCD}$ & 1.68 (0.18) CDE & $1.31(0.17) \mathrm{E}$ \\
\hline Urea & 1.64 (0.15) CDE & $2.61(0.20) \mathrm{ABC}$ & $2.27(0.15) \mathrm{ABCDE}$ & 1.96 (0.14) BCDE & 1.74 (0.16) CDE & $1.26(0.10) \mathrm{E}$ \\
\hline
\end{tabular}

Table 6. Responses of $\delta^{15} \mathrm{~N}(\%)$ of competing vegetation to fertilization treatments and time. Table entries are means ( \pm standard error of the mean) for each fertilizer treatment $\times$ time combination $(n=8)$. Means throughout the table marked with the same letters did not differ significantly $(\alpha=0.05)$.

\begin{tabular}{ccccccc}
\hline \multirow{2}{*}{$\begin{array}{c}\text { Fertilizer } \\
\text { Treatment }\end{array}$} & $\mathbf{5}$ & $\mathbf{5}$ Time (Months) \\
\cline { 2 - 7 } & $\mathbf{0}$ & $\mathbf{1}$ & $\mathbf{2}$ & $\mathbf{3}$ & $\mathbf{6}$ & $\mathbf{1 2}$ \\
\hline Control & $-0.05(0.28) \mathrm{GH}$ & $4.55(1.65) \mathrm{GH}$ & $9.59(6.79) \mathrm{FGH}$ & $13.83(11.90) \mathrm{EFGH}$ & $1.71(0.78) \mathrm{GH}$ & $-0.09(0.44) \mathrm{GH}$ \\
Agrotain & $-0.20(0.30) \mathrm{GH}$ & $216.68(16.48) \mathrm{A}$ & $207.53(11.30) \mathrm{AB}$ & $175.50(13.73) \mathrm{AB}$ & $87.61(15.46) \mathrm{D}$ & $82.69(13.05) \mathrm{DE}$ \\
Arborite & $-0.68(0.36) \mathrm{H}$ & $217.34(13.66) \mathrm{A}$ & $204.85(8.14) \mathrm{AB}$ & $166.11(11.84) \mathrm{ABC}$ & $73.53(9.26) \mathrm{D}$ & $73.85(12.55) \mathrm{DEF}$ \\
ESN & $-0.18(0.32) \mathrm{GH}$ & $94.32(13.27) \mathrm{D}$ & $178.98(13.06) \mathrm{AB}$ & $207.63(14.27) \mathrm{AB}$ & $111.89(7.21) \mathrm{CD}$ & $76.62(7.50) \mathrm{DE}$ \\
Urea & $0.51(0.62) \mathrm{GH}$ & $212.59(11.03) \mathrm{A}$ & $201.62(7.12) \mathrm{AB}$ & $153.89(18.87) \mathrm{BC}$ & $77.51(14.62) \mathrm{D}$ & $62.69(3.61) \mathrm{DEFG}$ \\
\hline
\end{tabular}


Table 7. Responses of $\delta^{15} \mathrm{~N}$ (\%) of litter to fertilization treatments and time. Table entries are means ( \pm standard error of the mean) for each fertilizer treatment $\times$ time combination $(n=8)$. Means throughout the table marked with the same letters did not differ significantly $(\alpha=0.05)$.

\begin{tabular}{ccccccc}
\hline \multirow{2}{*}{$\begin{array}{c}\text { Fertilizer } \\
\text { Treatment }\end{array}$} & $\mathbf{5}$ & \multicolumn{4}{c}{ Time (Months) } \\
\cline { 2 - 7 } & $\mathbf{0}$ & $\mathbf{1}$ & $\mathbf{2}$ & $\mathbf{3}$ & $\mathbf{6}$ & $\mathbf{1 2}$ \\
\hline Control & $0.55(1.98) \mathrm{E}$ & $7.46(3.75) \mathrm{E}$ & $6.81(1.00) \mathrm{E}$ & $31.63(27.77) \mathrm{DE}$ & $0.05(1.65) \mathrm{E}$ & $-1.81(0.23) \mathrm{E}$ \\
Agrotain & $-0.59(0.84) \mathrm{E}$ & $120.29(2.12) \mathrm{AB}$ & $137.67(19.53) \mathrm{A}$ & $134.19(19.15) \mathrm{AB}$ & $121.74(8.77) \mathrm{AB}$ & $99.41(8.41) \mathrm{ABC}$ \\
Arborite & $-0.64(0.92) \mathrm{E}$ & $106.38(20.93) \mathrm{ABC}$ & $116.63(14.33) \mathrm{AB}$ & $114.04(2.65) \mathrm{AB}$ & $102.20(5.67) \mathrm{AB}$ & $104.59(10.02) \mathrm{AB}$ \\
ESN & $0.38(1.22) \mathrm{E}$ & $42.45(2.80) \mathrm{CDE}$ & $113.47(18.67) \mathrm{AB}$ & $139.49(12.74) \mathrm{AB}$ & $118.30(10.64) \mathrm{AB}$ & $80.12(15.99) \mathrm{BCD}$ \\
Urea & $0.61(1.78) \mathrm{E}$ & $112.33(17.78) \mathrm{AB}$ & $123.58(21.63) \mathrm{AB}$ & $131.25(7.59) \mathrm{AB}$ & $110.21(11.53) \mathrm{AB}$ & $90.68(10.17) \mathrm{ABCD}$ \\
\hline
\end{tabular}

Fertilizer $\mathrm{N}$ recovery from competing vegetation and litter were determined as follows. First, sample $\mathrm{N}$ contents were determined by multiplying the measured $\mathrm{N}$ concentration of each sample by the average dry mass of its vegetation or litter sub-samples collected from each $0.0625 \mathrm{~m}^{2}$ sub-sampling area. Second, reported $\delta^{15} \mathrm{~N}$ values for each vegetation or litter sample at each sampling time were mathematically reverted to ${ }^{15} \mathrm{~N}:{ }^{14} \mathrm{~N}$ values via simple rearrangement of the equation used for calculating $\delta^{15} \mathrm{~N}$. Third, total mass of ${ }^{15} \mathrm{~N}$ in each vegetation or litter sample was determined based upon the calculated sample $\mathrm{N}$ content and ${ }^{15} \mathrm{~N}:{ }^{14} \mathrm{~N}$ of each sample. Fourth, values for total mass of ${ }^{15} \mathrm{~N}$ in each competing vegetation or litter sample collected at each sampling time from areas surrounding unfertilized control trees were assumed to be representative of ${ }^{15} \mathrm{~N}$ natural abundance in competing vegetation or litter, respectively, at each sampling time; consequently, these values were averaged for each ecosystem component at each sampling time and subtracted from the calculated total mass ${ }^{15} \mathrm{~N}$ per sample of vegetation and litter samples taken from areas surrounding fertilized crop trees at each sampling time to yield an estimate of the mass of fertilizerderived ${ }^{15} \mathrm{~N}$ per sample for each competing vegetation or litter sample. Fifth, the resulting values were then divided by $0.0625 \mathrm{~m}^{2}$ and multiplied by $100 \mathrm{~m}^{2}$ to yield estimates of fertilizer-derived ${ }^{15} \mathrm{~N}$ contents of the competing vegetation and litter present within the $100 \mathrm{~m}^{2}$ area surrounding each crop tree to which fertilizer treatments were applied. Finally, the estimated mass of fertilizer-derived ${ }^{15} \mathrm{~N}$ present in the competing vegetation or litter of the $100 \mathrm{~m}^{2}$ areas surrounding each fertilized crop tree at each sampling time was divided by the mass of ${ }^{15} \mathrm{~N}$ initially applied via each fertilizer treatment and expressed as a percentage to produce an estimate of FNR from the competing vegetation and litter components surrounding each fertilized black walnut crop tree at each sampling time.

The experiment was designed and executed as a randomized complete block design $(n=8)$. Data for each response variable were analyzed using ANOVA $(p<0.05)$ followed by Tukey's Multiple Pairwise Comparison $(\alpha=0.05)$ to detect significant means separations within significant model factors (SAS 9.4, SAS Institute, Inc., Cary, NC, USA). Data for each ecosystem component (i.e., black walnut crop tree leaves, competing vegetation and litter) were analyzed separately, with fertilizer treatment, sampling time, and fertilizer treatment $\times$ sampling time included as model factors for each ecosystem component.

\section{Results}

\subsection{Crop Tree Leaves}

Black walnut leaf dry mass was affected by fertilizer treatment $(p=0.0384)$ and time ( $p<0.0001$; Tables 1-3). Arborite applications resulted in greater crop tree leaf mass relative to Agrotain (Table 2). Crop tree leaf area was not affected by fertilizer treatment $(p=0.0710$; Tables 1 and 2), although it was influenced by time $(p<0.0001$; Tables 1 and 3$)$. Mean leaf area increased over time following fertilization compared to time zero sampling (Table 3). Crop tree leaf $\mathrm{N}$ concentrations were affected by both fertilizer treatment and time $(p=0.0301$ and $<0.0001$, respectively; Tables 1-3). Application of Arborite resulted in greater leaf $\mathrm{N}$ concentrations relative to unfertilized controls (Table 2), and leaf $\mathrm{N}$ concentrations increased over time (Table 3). However, leaf $\mathrm{N}$ content of crop trees was unaffected by either fertilization or sampling time (overall model $p=0.0908$; Tables 1-3). Black walnut 
leaf $\delta^{15} \mathrm{~N}$ was influenced by an interaction between fertilization and time $(p=0.0026$; Tables 1 and 4). No differences in $\delta^{15} \mathrm{~N}$ were observed among the time zero reference leaves across treatments or between the time zero reference leaves of all treatments and leaves collected from the unfertilized control trees throughout the study. Then, one month after fertilization, Agrotain applications resulted in the most rapid elevation of leaf $\delta^{15} \mathrm{~N}$ relative to control trees or time zero reference leaves. At two months after fertilization, leaf $\delta^{15} \mathrm{~N}$ was elevated in crop trees of both the Agrotain and Arborite treatments relative to unfertilized controls. Then, three months after fertilization, $\delta^{15} \mathrm{~N}$ was elevated in leaves of all fertilized crop trees relative to controls. Only ESN exhibited a slow release pattern, with lower leaf $\delta^{15} \mathrm{~N}$ values observed early in the season that increased over time before peaking at three months, and declining thereafter (Table 4). Neither fertilizer product nor time affected recovery efficiency of applied $\mathrm{N}$ in leaves of black walnut crop trees (overall model $p=0.6996$; Tables 1-3).

\subsection{Competing Vegetation}

Although dry mass of competing vegetation was not affected by fertilizer treatments ( $p=0.6466$; Tables 1 and 2), it was significantly influenced by sampling time $(p<0.0001$; Tables 1 and 3). Interestingly, the mass of competing vegetation was greater in month zero than in months three or six, and it was greater in months 2, 3, and 12 than in month 6 (Table 3$)$. The $\mathrm{N}$ concentration of competing vegetation varied in response to an interaction between fertilization and time ( $p=0.0112$; Tables 1 and 5). No differences in $\mathrm{N}$ concentrations of competing vegetation were observed between time zero reference samples or between control treatment samples throughout the study. One month after fertilization, $\mathrm{N}$ concentrations of competing vegetation in the Agrotain and Arborite treatments had increased above their time zero reference values and unfertilized controls. By the sixth and third months, respectively, $\mathrm{N}$ concentrations of competing vegetation in the Agrotain and Arborite treatments dropped significantly below their first month spikes to levels commensurate with their time zero control values. Although $\mathrm{N}$ contents of competing vegetation were not affected by fertilization ( $p=0.0751$; Tables 1 and 2$)$, they were significantly influenced by sampling time ( $p<0.0001$; Tables 1 and 3$)$.

Competing vegetation $\delta^{15} \mathrm{~N}$ was affected by an interaction between fertilization and sampling time $\left(p<0.0001\right.$; Tables 1 and 6). Competing vegetation $\delta^{15} \mathrm{~N}$ in all fertilized treatments was elevated above that of unfertilized control treatments one month after fertilization, although the $\delta^{15} \mathrm{~N}$ associated with ESN remained lower than that observed in all other treatments.

Agrotain, Arborite, and urea showed higher $\delta^{15} \mathrm{~N}$ values, which reflected rapid availability and uptake before values peaked at 3 months after application and then declined thereafter (Table 6). Although fertilizer treatment did not affect FNR efficiency of applied $\mathrm{N}$ in competing vegetation ( $p=0.8375$; Tables 1 and 2), FNR of applied $\mathrm{N}$ in competing vegetation was influenced by time ( $p<0.0001$; Tables 1 and 3). Fertilizer $\mathrm{N}$ recovery from competing vegetation was highest at one month after fertilization and decreased over time (Table 3).

\subsection{Litter}

Although the dry mass of forest floor litter was unaffected by fertilization $(p=0.9419$; Tables 1 and 2), it significantly increased over time $(p<0.0001$; Tables 1 and 3) during the first three months after fertilization (Table 3). Neither fertilization treatment nor sampling time influenced the $\mathrm{N}$ concentration of forest floor litter (overall model $p=0.0655$; Tables 1-3). Although the $\mathrm{N}$ content of forest floor litter was unaffected by fertilizer treatment ( $p=0.7472$; Tables 1 and 2), it was influenced by time $(p<0.0001$; Tables 1 and 3$)$. Litter $\mathrm{N}$ content was higher in months zero and six than in the first three months (Table 3). An interaction between fertilization treatment and time was observed for $\delta^{15} \mathrm{~N}$ of forest floor litter $(p<0.0001$; Tables 1 and 7), with only the ESN treatment showing evidence of the delayed incorporation of fertilizer-derived ${ }^{15} \mathrm{~N}$ into litter (Table 7). Neither fertilizer 
type nor sampling time influenced FNR in forest floor litter (overall model $p=0.9112$; Tables 1-3).

\section{Discussion}

\subsection{Study Questions and Rationale}

This study evaluated differences in the uptake and partitioning of applied fertilizer $\mathrm{N}$ in aboveground ecosystem components in a mid-rotation walnut plantation. Overall, three urea-based EEF products (Agrotain Ultra, Arborite EC, Agrium ESN), uncoated urea, and an unfertilized control were examined. The goal was to improve our understanding of the fate of applied fertilizers so as to better inform fertilizer recommendations for plantation forestry. Key questions addressed by this study were: (1) whether there were differences in $\mathrm{N}$ concentrations of aboveground ecosystem components in response to fertilizer treatments; (2) whether there were differences in FNR between uncoated urea and EEFs; and (3) whether there were differences in aboveground component $\delta^{15} \mathrm{~N}$ levels between fertilizer products, with higher values reflecting higher levels of nutrient availability and uptake. The overall objective was to improve our understanding of the fate of applied $\mathrm{N}$ in both conventional and EEF forms so as to enhance FNR and FNUE in mid-rotation black walnut plantations. Our study results in relation to findings of other studies are discussed below.

\subsection{Nitrogen Concentrations and $\delta^{15} \mathrm{~N}$ Values in Ecosystem Components}

There were no significant fertilizer main effects on ecosystem component $\mathrm{N}$ concentrations except in crop tree leaves, where Arborite applications resulted in higher $\mathrm{N}$ concentrations compared to unfertilized controls (Tables 2 and 5). Although a complex interaction was observed for $\mathrm{N}$ concentrations in competing vegetation, no differences were observed between fertilized treatments at a given sampling time (Tables 1 and 5). Foliar $\delta^{15} \mathrm{~N}$ values are valuable tools to assess plant $\mathrm{N}$ uptake patterns and characterize $\mathrm{N}$ cycling across different ecosystems and ecosystem components [18]. In our study, the release pattern of ESN resulted in lower $\delta^{15} \mathrm{~N}$ values in crop tree leaves early in the season, which increased gradually during the three months after application (Table 4). A similar pattern was observed for competing vegetation, with decreases observed following the peak values observed at three months after application (Table 6). This pattern is consistent with the slow release of $\mathrm{N}$ from the applied ESN, whereas the release patterns observed for other fertilizers did not differ from uncoated urea in this study (Tables 4 and 6). However, the slow-release pattern exhibited by ESN and not the others in this study differs from other studies that have suggested slow-release patterns for all [11]. The slow-release behavior of ESN observed in this study has the potential to better facilitate synchronization of fertilizer $\mathrm{N}$ release with plant $\mathrm{N}$ demand compared to the other products studied. Fertilization with Agrotain, Arborite, and urea resulted in generally higher $\delta^{15} \mathrm{~N}$ values in ecosystem components more rapidly after application, suggesting more rapid increases in nutrient availability.

\subsection{Fertilizer $N$ Recovery in Ecosystem Components}

There were no significant differences in aboveground ecosystem FNR rates between fertilizer treatments, with total aboveground FNR in the components measured ranging from $9.59 \%$ in Argrotain to $10.87 \%$ in urea (Table 2). These results contrast with results of other studies where FNR was significantly higher for all EEFs compared to urea, but with no differences observed between EEFs [11]. In the [11] study, the total ecosystem FNR ranged from 81.9 to $84.2 \%$ for EEFs compared to $65.2 \%$ for urea. [11] found that the largest amount of fertilizer-derived $\mathrm{N}$ for all treatments was found in the loblolly pine crop trees $(38.5 \%-49.9 \%$ for EEFs and $34.8 \%$ for urea) and in the soil surrounding the crop trees (30.6\%-38.8\% for EEFs and $28.4 \%$ for urea). In our study, total aboveground FNR ranged from about $16 \%$ at one month after fertilization to about $3 \%$ at 12 months after fertilization, reflecting potential resorption and remobilization of $\mathrm{N}$ to woody tissues and roots. The large amounts of applied fertilizer unaccounted for in this study likely resulted 
from volatilization and leaching as found in other studies [11,19-21] or remobilization and storage in woody tissues and soil compartments [11]. Ref. [11] found that higher $\mathrm{N}$ losses observed for applied urea $(26 \%-49 \%)$ were due primarily to $\mathrm{NH}_{3}$ volatilization. Other studies in pine plantations in the southeastern United States have reported similarly high $\mathrm{N}$ losses through $\mathrm{NH}_{3}$ volatilization (more than $25 \%$ ) after urea fertilization, with lower losses noted for various EEF products [21-23].

Although urea is the most commonly used $\mathrm{N}$ fertilizer in southern forestry due to its high $\mathrm{N}$ content and low cost per unit of applied $\mathrm{N}[24,25]$, further pilot and operational scale studies are needed to help understand and address the large $\mathrm{N}$ losses through ammonia $\left(\mathrm{NH}_{3}\right)$ volatilization [11,19-21]. Additionally, there remains a need to refine coating technology to minimize $\mathrm{N}$ losses from EEF products, as evidenced by the low FNR rates observed in this study. Volatilization, combined with leaching and denitrification, reduces the amount of fertilizer $\mathrm{N}$ remaining in the system and may decrease fertilizer $\mathrm{N}$ availability for plant uptake and hence FNUE [26,27]. Additionally, the greater FNR observed for competing vegetation in this study suggests competing vegetation pre-empted uptake of applied fertilizer to the detriment of target black walnut trees. The demonstrated stronger sink strength of competing vegetation compared with black walnut trees has potential to negatively affect FNUE, suggesting that fertilization should be conducted in combination with weed control to benefit plantation productivity $[28,29]$. In particular, weed control kills non-target vegetation, creates space, and releases nutrients and moisture to promote rapid growth and can reduce rotation length of target crops [30-34]. Furthermore, [34] reported that intensive management of hardwood plantations in Spain where chemical weed control is used in combination with irrigation and fertilization to stimulate rapid growth and reduce plantation rotation length (typically about 50-60 years) by half (to about 20-25 years), thus corroborating the need to combine weed control practices with fertilization to accelerate hardwood plantation growth and productivity $[4,22,30,32]$.

\section{Conclusions}

This study examined the short-term impacts and fates of urea-based EEFs compared with uncoated urea in a mid-rotation black walnut stand in Indiana, USA. Fertilizer N recovery did not vary by fertilizer treatment, but competing vegetation was a strong sink for applied $\mathrm{N}$ with the potential to negatively impact FNUE and growth of target trees. The low FNR rates observed in this study corroborate results of some studies but differ from others where more than $90 \%$ of applied $\mathrm{N}$ was recovered. Although not quantified, storage in woody tissues and soil compartments, volatilization, and leaching likely contributed to the low FNR rates observed in our study. This highlights the need for nutrient budget studies to comprehensively quantify native contributions and fate and transport of applied fertilizers in aboveground and belowground ecosystem components to enable accurate accounting of what translates to productivity and losses from the system. Only ESN exhibited a slowrelease pattern, as indicated by lower $\delta^{15} \mathrm{~N}$ values early in the season, which increased over time until 3 months after application before declining thereafter. Fertilization with Agrotain, Arborite, and Urea resulted in higher $\delta^{15} \mathrm{~N}$ values in ecosystem components more rapidly after application, suggesting rapid increases in nutrient availability and little to no slow-release behavior. Based on patterns of $\mathrm{N}$ uptake by competing vegetation observed in this study, it appears that intensive plantation management practices where weed control is combined with fertilization could improve the availability of applied $\mathrm{N}$ for crop trees.

Author Contributions: Conceptualization: J.L.S., D.F.J.; Data curation: J.L.S.; Formal analysis: J.L.S.; Funding acquisition: D.F.J.; Investigation: J.L.S., D.F.J; Methodology: J.L.S., D.F.J.; Project administration: J.L.S., D.F.J.; Resources: J.L.S., D.F.J.; Software: J.L.S.; Supervision: J.L.S., D.F.J.; Validation: J.L.S.; Visualization: J.L.S.; Writing—original draft: J.L.S., F.K.S.; Writing—review and editing: J.L.S., F.K.S., D.F.J. All authors have read and agreed to the published version of the manuscript.

Funding: Funding for this project was provided by the National Science Foundation Center for Advanced Forestry Systems award number IIP-1031473 and the Walnut Council Foundation. 
Institutional Review Board Statement: Not applicable.

Informed Consent Statement: Not applicable.

Data Availability Statement: The data presented in this study are available upon request to the corresponding author.

Acknowledgments: The authors thank Justin Schmal, Derek Heacock, Miranda Vogel, Juan Oliet, Shucai Zeng, Kyle Rose, and Brian Strahm for their technical assistance and expertise.

Conflicts of Interest: The authors declare no conflict of interest.

\section{References}

1. Michler, C.; Woeste, K.E.; Pijut, P.M. Black walnut. In Genome Mapping and Molecular Breeding in Plants; Forest Trees; Kole, C., Ed.; Springer: Berlin, Germany, 2007; Volume 7, pp. 189-198.

2. Williams, R.D.; Juglans nigra, L. Black walnut. In Silvics of North America, Hardwoods. Agriculture Handbook 654; Burns, R.M., Honkala, B.H., Eds.; U.S. Department of Agriculture, Forest Service: Washington, DC, USA, 1990; Volume 2, pp. 391-399.

3. Noweg, T.A.; Kurtz, W.B. Eastern Black Walnut Plantations: An Economically Viable Option for Conservation Reserve Lands within the Corn Belt. North. J. Appl. For. 1987, 4, 158-160. [CrossRef]

4. Pedlar, J.H.; McKenney, D.W.; Fraleigh, S. Planting black walnut in southern Ontario: Mid-rotation assessment of growth, yield, and silvicultural treatments. Can. J. For. Res. 2006, 36, 495-504. [CrossRef]

5. Shifley, S.R. The black walnut resource in the United States. In Black Walnut in a New Century, Proceedings of the 6th Walnut Council Research Symposium, Lafayette, Indiana, 25-28 July 2004; Michler, C.H., Pijut, P.M., Van Sambeek, J.W., Coggeshall, M.V., Seifert, J., Woeste, K., Overton, R., Ponder, F., Jr., Eds.; U.S. Department of Agriculture, Forest Service, North Central Research Station: St. Paul, MN, USA, 2004; 188p.

6. Jacobs, D.F.; Ross-Davis, A.L.; Davis, A.S. Establishment success of conservation tree plantations in relation to silvicultural practices in Indiana, USA. New For. 2004, 28, 23-36. [CrossRef]

7. Albaugh, T.A.; Allen, H.L.; Fox, T.R. Historical patterns of forest fertilization in the southern United States from 1969 to 2004. South. J. Appl. For. 2007, 31, 129-137. [CrossRef]

8. Goodman, R.C.; Oliet, J.A.; Sloan, J.L.; Jacobs, D.F. Nitrogen fertilization of black walnut (Juglans nigra L.) during plantation establishment. Physiology of production. Eur. J. For. Res. 2013, 133, 153-164. [CrossRef]

9. Bravo, K.; Marcolini, G.; Sorrenti, G.; Baldi, E.; Quartieri, M.; Toselli, M. Effect of time of application on nitrogen uptake, partitioning, and remobilization in walnut trees. J. Plant Nutr. 2017, 40, 719-725. [CrossRef]

10. Goodman, R.C.; Oliet, J.A.; Pardillo, G.; Jacobs, D.F. Nitrogen Fertilization of Black Walnut (Juglans nigra L.) During Plantation Establishment. Morphology and Production Efficiency. For. Sci. 2013, 59, 453-463. [CrossRef]

11. Raymond, J.E.; Fox, T.R.; Strahm, B.D.; Zerpa, J. Ammonia volatilization following nitrogen fertilization with enhanced efficiency fertilizers and urea in loblolly pine (Pinus taeda L.) plantations of the southern United States. For. Ecol. Manag. 2016, 376, 247-255. [CrossRef]

12. Raymond, J.E.; Fox, T.R.; Strahm, B.D. Understanding the Fate of Applied Nitrogen in Pine Plantations of the Southeastern United States Using 15N Enriched Fertilizers. Forests 2016, 7, 270. [CrossRef]

13. Sloan, J.L.; Uscola, M.; Jacobs, D.F. Nitrogen recovery in planted seedlings, competing vegetation, and soil in response to fertilization on a boreal mine reclamation site. For. Ecol. Manag. 2016, 360, 60-68. [CrossRef]

14. Trenkel, M.E. Slow-and Controlled-Release and Stabilized Fertilizers: An Option for Enhancing Nutrient Use Efficiency in Agriculture; International Fertilizer Industry Association (IFA): Paris, France, 2010.

15. Azeem, B.; KuShaari, K.; Man, Z.B.; Basit, A.; Thanh, T.H. Review on materials \& methods to produce controlled release coated urea fertilizer. J. Control. Release 2014, 181, 11-21. [CrossRef]

16. Hauck, R.D. Slow release and bio-inhibitor-amended nitrogen fertilizers. In Fertilizer Technology and Use, 3rd ed.; Engelstad, O.P., Ed.; SSSA: Madison, WI, USA, 1985; pp. 293-322.

17. Zellers, C.E.; Saunders, M.R.; Morrissey, R.C.; Shields, J.M.; Bailey, B.G.; Dyer, J.; Cook, J. Development of allometric leaf area models for intensively managed black walnut (Juglans nigra L.). Ann. For. Sci. 2012, 69, 907-913. [CrossRef]

18. Kahmen, A.; Wanek, W.; Buchmann, N. Foliar $\delta^{15} \mathrm{~N}$ values characterize soil $\mathrm{N}$ cycling and reflect nitrate or ammonium preference of plants along a temperate grassland gradient. Oecologia 2008, 156, 861-870. [CrossRef]

19. Zhang, X.; Mauzerall, D.L.; Davidson, E.A.; Kanter, D.R.; Cai, R. The economic and environmental consequences of implementing nitrogen-efficient technologies and management practices in agriculture. J. Environ. Qual. 2015, 44, 312-324. [CrossRef] [PubMed]

20. Cabrera, M.L.; Kissel, D.E.; Vaio, N.; Craig, J.R.; Rema, J.A.; Morris, L.A. Loblolly Pine Needles Retain Urea Fertilizer that Can Be Lost as Ammonia. Soil Sci. Soc. Am. J. 2005, 69, 1525-1531. [CrossRef]

21. Elliot, J.R.; Fox, T.R. Ammonia Volatilization Following Fertilization with Urea or Ureaform in a Thinned Loblolly Pine Plantation. Soil Sci. Soc. Am. J. 2014, 78, 1469-1473. [CrossRef]

22. Zerpa, J.L.; Fox, T.R. Controls on volatile $\mathrm{NH}_{3}$ losses from loblolly pine plantations fertilized with urea in the southeast USA. Soil Sci. Soc. Am. J. 2011, 75, 257-266. [CrossRef] 
23. Kissel, D.; Cabrera, M.; Vaio, N.; Craig, J.; Rema, J.; Morris, L. Forest Floor Composition and Ammonia Loss from Urea in a Loblolly Pine Plantation. Soil Sci. Soc. Am. J. 2009, 73, 630-637. [CrossRef]

24. Allen, H.L. Forest fertilizers: Nutrient amendment, and productivity, and environmental impact. J. For. 1987, 85, 37-46.

25. Fox, T.R.; Allen, H.L.; Albaugh, T.J.; Rubilar, R.; Carlson, C.A. Tree Nutrition and Forest Fertilization of Pine Plantations in the Southern United States. South. J. Appl. For. 2007, 31, 5-11. [CrossRef]

26. Shrestha, R.K.; Strahm, B.D.; Sucre, E.B. Nitrous Oxide Fluxes in Fertilized Pinus taeda L. Plantations across a Gradient of Soil Drainage Classes. J. Environ. Qual. 2014, 43, 1823-1832. [CrossRef]

27. Aust, W.M.; Blinn, C.R. Forestry best management practices for timber harvesting and site preparation in the eastern United States: An overview of water quality and productivity research during the past 20 years (1982-2002). Water Air Soil Pollut. Focus 2004, 4, 5-36. [CrossRef]

28. Pope, P.E.; Holt, H.A.; Chaney, W.R. Interaction of nitrogen fertilization and chemical weed control on four-year volume growth of a black walnut plantation. In Black Walnut for the Future; USDA FS Gen. Tech. Rep. NC-74; North Central Forest Experiment Station: St. Paul, MN, USA, 1982.

29. Jacobs, D.F.; Salifu, K.F.; Seifert, J.R. Growth and nutritional response of hardwood seedlings to controlled-release fertilization at outplanting. For. Ecol. Manag. 2005, 214, 28-39. [CrossRef]

30. Von Althen, F.W. Revitalizing a black walnut plantation through weed control and fertilization. For. Chron. 1985, 61, 71-74. [CrossRef]

31. Cogliastro, A.; Gagnon, D.; Coderre, D.; Bhéreur, P. Response of seven hardwood tree species to herbicide, rototilling, and legume cover at two southern Quebec plantation sites. Can. J. For. Res. 1990, 20, 1172-1182. [CrossRef]

32. Talbert, C.; Marshall, D. Plantation productivity in the Douglas-fir region under intensive silvicultural practices: Results from research and operations. J. For. 2005, 103, 65-70.

33. Chien, S.H.; Prochnow, L.I.; Cantarella, H. Recent developments of Fertilizer Production and Use to Improve Nutrient Efficiency and Minimize Environmental Impacts. In Advances in Agronomy 102; Elsevier Inc.: Newark, NJ, USA, 2009; pp. $267-322$.

34. López-Díaz, M.; Benítez, R.; Moreno, G. How do management techniques affect carbon stock in intensive hardwood plantations? For. Ecol. Manag. 2017, 389, 228-239. [CrossRef] 